\title{
New-Onset Perioperative Atrial Fibrillation Is Associated with Increased All-Cause Mortality in Elderly Patients Undergoing Total Knee and Hip Replacements
}

\author{
Ben Varon ${ }^{\mathrm{a}}$ Leonid Kandel ${ }^{\mathrm{b}}$ Gurion Rivkin ${ }^{\mathrm{b}}$ David Leibowitz ${ }^{\mathrm{a}}$ \\ ${ }^{a}$ Department of Cardiology, Hadassah-Hebrew University Medical Center, Jerusalem, Israel; b Department of \\ Orthopedics, Hadassah-Hebrew University Medical Center, Jerusalem, Israel
}

\section{Keywords}

Elderly · Atrial fibrillation · Hip replacement · Knee

replacement

\begin{abstract}
Introduction: Total hip arthroplasty (THA) and total knee arthroplasty (TKA) are common surgeries performed in elderly patients with osteoarthritis. Limited data address the clinical significance of perioperative atrial fibrillation (AF) in these patients. This study aimed to determine whether preexisting or new-onset AF is associated with increased 1-year all-cause mortality rates in the elderly population. Methods: 280 patients over the age of 60 undergoing THA or TKA with perioperative AF and 280 control-matched patients were retrospectively identified, and their files reviewed. The primary end point was 1-year all-cause mortality from the date of the surgery. Results: Of the 280 patients with perioperative AF, 37 had new-onset AF with a 1-year all-cause mortality rate of $10.8 \%$. This mortality was significantly higher in patients with new-onset AF compared to patients without AF or patients with previous AF $(10.8 \%$ vs. $1.1 \%$ and $2.5 \%$, respectively; $p=0.005)$. On multivariate analysis, this difference remained significant after adjustment for risk factors associ-
\end{abstract}

karger@karger.com www.karger.com/ger

Karger $\stackrel{\text { ' }}{5}$

GOPEN ACCESS
(C) 2021 The Author(s)

Published by S. Karger AG, Basel

This is an Open Access article licensed under the Creative Commons Attribution-NonCommercial-4.0 International License (CC BY-NC) (http://www.karger.com/Services/OpenAccessLicense), applicable to the online version of the article only. Usage and distribution for commercial purposes requires written permission. ated with all-cause mortality. Conclusions: One-year all-cause mortality in elderly patients undergoing TKA or THA is significantly increased in the patients that develop new postoperative AF. These patients warrant increased clinical surveillance following surgery.

(C) 2021 The Author(s)

Published by S. Karger AG, Basel

\section{Introduction}

Osteoarthritis (OA) is a chronic degenerative disease of the joints [1]. An estimated 27 million adults in the United States suffer from OA, which is more prevalent with increasing age $[1,2]$. Total joint arthroplasties for knees and hips are mainstay forms of treatment for severe, refractory OA causing debilitating symptoms [3]. The treatment is efficient, cost-effective, improves quality of life, and usage rates are expected to rise significantly in the near future, particularly given the aging of the population [3, 4]. Different studies have assessed mortality in joint replacement surgeries, with a recent study also including younger patients reporting 1-year mortality rates of 0.9 and $1.1 \%$ for total knee arthroplasty (TKA) and total hip arthroplasty (THA), respectively [5]. Atrial 
fibrillation (AF) is the most common arrhythmia in the general population with lifetime risk estimations as high as $22.7 \%$ in males and $21.6 \%$ in females, above the age of 80 [6]. Clinical consequences of AF include decreased cardiac output and risk of thromboembolism, and the presence of AF is associated with increased mortality (as high as 1.5-1.9 times that of the general population) [7]. $\mathrm{AF}$ has also been associated with an increased 30-day mortality rate (6.4\%) after noncardiac surgery [8].

Data concerning AF in elective orthopedic surgeries is somewhat limited. One study found that patients with preexisting AF undergoing TKA suffered more postoperative complications such as bleeding, infections, and stroke, but this study did not address mortality or the implications of new-onset AF [9]. Another study found that new-onset AF after TKA and THA was associated with a greater risk for stroke within 1 year after the surgery but did not address mortality either [10]. Finally, a recent study found that patients undergoing hip arthroplasty have increased 1-year mortality; however, this study ruled out patients with preexisting AF and did not specify whether the operation was elective or following a hip fracture [11].

As both $\mathrm{OA}$ and $\mathrm{AF}$ are disorders whose prevalence increases with age, patients requiring TKA or THA frequently have a history of preoperative AF. As so, more information regarding the clinical significance of preexisting or new-onset AF in this setting is needed. The aim of the current study was to investigate whether preoperative or newly diagnosed AF among elderly patients undergoing THA/ TKA is predictive of 1-year all-cause mortality.

\section{Methods}

All patients over the age of 60 years who underwent TKA or THA were retrospectively identified using the institutional database. All patients were operated on utilizing the same operative protocol and same joint prosthesis. Patient charts from January 2007 to December 2016 were reviewed. Exclusion criteria included revision TKA or THA, THA as a treatment for femoral neck fractures and bilateral synchronous joint replacements. Patient charts were reviewed for preoperative history, medication use, operative details, and type (paroxysmal AF [PAF], chronic AF [CAF], or new onset) \& timing of AF (pre-op, post-op, or both), as well as inhospital treatment of AF (if needed). Given the different potential clinical implications of CAF versus PAF prior to surgery as well as AF appearing only following surgery, different analyses were performed for type and timing of AF [12]. New-onset AF was defined as AF demonstrated postoperatively in patients with no previous clinical history of AF. All-cause mortality was defined as death of any cause within the first year following date of surgery and was identified using the national Ministry of Health database. The study was approved by the institutional review board.
A total of 280 patients with pre/postoperative AF were identified and assessed, along with a group of 280 control patients. The control group included TKA/THA patients with no documented AF. Two experienced investigators manually matched the AF group for age (within 5 years), gender, similar operation dates, and type of surgery (TKA or THA).

Comparisons between groups were performed using 2-sample $t$ tests or the Mann-Whitney nonparametric test for continuous variables and $\chi^{2}$ or Fisher's exact test for categorical variables. Variables noted to be significant on univariate analysis were evaluated in a multivariate model using stepwise logistic regression analysis. Odds ratios (ORs) are reported with $95 \%$ confidence intervals. All statistical tests were 2 sided, and a $p$ value of $<0.05$ was considered statistically significant. The statistical program used for the analyses was the Statistical Product and Service Solutions (SPSS).

\section{Results}

A total of 560 patients were included in study, half of whom had perioperative AF. Clinical characteristics of the 2 groups are depicted in Table 1 . The mean age was approximately 75 years for both groups. About $60 \%$ of the patients in both groups were females. Sixty-three percent of the patients had TKAs, and 37\% had THAs. All surgeries were performed in an elective setting.

At 1-year follow-up, a total of 13 patients (2.3\%) had died: 10 in the AF group (3.6\%) and 3 in the control group (1.1\%). A comparison between the survivors and nonsurvivors can be found in Table 2. The difference in mortalities between the groups was statistically significant on univariate analysis $(p=0.049)$. Other variables associated with all-cause mortality were as follows: age $(p=0.025)$, high ASA score (3-4) $(p=0.025)$, general anesthesia $(p=$ $0.048)$, chronic heart failure $(\mathrm{CHF})(p=0.003)$, and chronic renal failure (CRF) $(p=0.001)$, as can be seen in online suppl. Table 1 (for all online suppl. material, see www.karger.com/doi/10.1159/000514482), along with corresponding likelihood ratios. The $t$ value for age was -2.248 . When these variables significant on univariate analysis were entered into a multivariate analysis, only the following variables were found to be significantly associated with all-cause mortality: age, general anesthesia, $\mathrm{CHF}$, and CRF, as demonstrated in Table 3.

A subgroup analysis examining the different clinical types of AF was performed. The characteristics of the AF group are demonstrated in Table 4. On univariate analysis of the AF group, the following variables were found to be significantly associated with all-cause mortality: age $(p=0.05)$, CRF $(p=0.005)$, type of AF (new and CAF) $(p=0.004)$, timing of AF onset (postoperative) $(p=0.014)$, treatment with rate control $(p=0.003)$, and treatment 
Table 1. Study group characteristics

\begin{tabular}{|c|c|c|c|c|}
\hline Characteristic & $\begin{array}{l}\text { AF group } \\
(n=280)\end{array}$ & $\begin{array}{l}\text { Control group } \\
(n=280)\end{array}$ & $p$ value & $\begin{array}{l}\text { Combined } \\
(n=560)\end{array}$ \\
\hline \multicolumn{5}{|l|}{ Demographics } \\
\hline \multicolumn{5}{|l|}{ Sex, $n(\%)$} \\
\hline Male & $109(38.9)$ & $111(39.6)$ & \multirow{2}{*}{0.863} & $220(39.3)$ \\
\hline Female & $171(61.1)$ & $169(60.4)$ & & $340(60.7)$ \\
\hline Age (mean) & 75.21 & 74.80 & 0.489 & 75.01 \\
\hline BMI (mean) & 29.62 & 29.35 & 0.57 & 29.49 \\
\hline \multicolumn{5}{|l|}{ Operative factors } \\
\hline \multicolumn{5}{|l|}{ ASA, $n(\%)$} \\
\hline $1-2$ & $130(46.4)$ & $210(75.0)$ & \multirow{2}{*}{$<0.001$} & $340(60.7)$ \\
\hline $3-4$ & $150(53.6)$ & $70(25.0)$ & & $220(39.3)$ \\
\hline \multicolumn{5}{|l|}{ Anesthesia type, $n(\%)$} \\
\hline Regional & $253(90.4)$ & $244(87.1)$ & \multirow{2}{*}{0.229} & $497(88.8)$ \\
\hline General & $27(9.6)$ & $36(12.9)$ & & $63(11.2)$ \\
\hline \multicolumn{5}{|l|}{ Operation type, $n(\%)$} \\
\hline THA & $105(37.5)$ & $104(37.1)$ & \multirow{2}{*}{0.93} & $209(37.3)$ \\
\hline TKA & $175(62.5)$ & $176(62.9)$ & & $351(62.7)$ \\
\hline \multicolumn{5}{|l|}{ Comorbidities, $n(\%)$} \\
\hline Diabetes mellitus & $61(21.8)$ & $63(22.5)$ & 0.839 & $124(22.1)$ \\
\hline Hypertension & $234(83.6)$ & $215(76.8)$ & 0.044 & $449(80.2)$ \\
\hline Ischemic heart disease & $72(25.7)$ & $55(19.6)$ & 0.086 & $127(22.7)$ \\
\hline $\mathrm{CHF}$ & $27(9.6)$ & $3(1.1)$ & $<0.001$ & $30(5.4)$ \\
\hline COPD & $21(7.5)$ & $19(6.8)$ & 0.743 & $40(7.1)$ \\
\hline CRF & $38(13.6)$ & $19(6.8)$ & 0.008 & $57(10.2)$ \\
\hline \multicolumn{5}{|l|}{ Chronic medications, $n(\%)$} \\
\hline Beta blockers & $143(51.1)$ & $114(40.7)$ & 0.014 & $257(45.9)$ \\
\hline Anticoagulation & $125(44.6)$ & $5(1.8)$ & $<0.001$ & $130(23.2)$ \\
\hline
\end{tabular}

AF, atrial fibrillation; ASA, American Society of Anesthesiologists; COPD, chronic obstructive pulmonary disease; THA, total hip arthroplasty; TKA, total knee arthroplasty; CHF, chronic heart failure; CRF, chronic renal failure. The values in bold are $p<0.05$.

with anticoagulation $(p=0.035)$ as demonstrated in online suppl. Table 2 . In a multivariate analysis of age, CRF, and AF type, only CRF and new-onset AF were found to be significantly associated with all-cause mortality with ORs of 7.64 and 9.95, respectively (Table 5). In similar fashion, a multivariate analysis of age, CRF, and AF timing was performed and demonstrated that CRF and postoperative AF are associated with ORs of 7.47 and 7.59, respectively. Patients with episodes of AF both prior to and following surgery did not have increased all-cause mortality.

An additional multivariate analysis of age, CRF, AF timing, treatment with anticoagulation, and treatment with rate control was performed. This analysis demonstrated that CRF and treatment with rate control were associated with increased mortality, as shown in online suppl. Table 3.

New-Onset Perioperative AF and Mortality in Arthroplasties

\section{Discussion}

This study demonstrated a significant association between new-onset postoperative $\mathrm{AF}$ in patients over the age of 60 undergoing TKA or THA and 1-year all-cause mortality. Previous studies have shown increased mortality and longer ICU stay in patients who developed postoperative AF after major noncardiac surgery $[8,13]$. Other recent studies demonstrated a significant association between post-op AF and increased 1-year mortality in patients undergoing hip fracture repair surgery and THA $[11,14]$. This study assessed patients undergoing hip or knee replacements in an elective setting and the effect of a new-onset $\mathrm{AF}$ on their all-cause mortality rate.

The 1-year all-cause mortality rate of $1.1 \%$ in the control group in this study is similar to the rate described in the current literature [5]. Age, CHF, and CRF are well- 
Table 2. A comparison between the deceased and alive patients in both groups (AF and control)

\begin{tabular}{lcc}
\hline Characteristic & $\begin{array}{l}\text { Deceased }- \\
\text { both groups } \\
(n=13)\end{array}$ & $\begin{array}{l}\text { Alive - } \\
\text { both groups } \\
(n=547)\end{array}$ \\
\hline $\begin{array}{c}\text { Demographics } \\
\text { Sex, } n(\%)\end{array}$ & \\
Male & $5(38.5)$ & $215(39.3)$ \\
Female & $8(61.5)$ & $332(60.7)$ \\
Age (mean) & 79.3 & 74.9 \\
BMI (mean) & 28 & 29.5 \\
Operative factors & & \\
ASA, $n(\%)$ & $4(30.8)$ & $336(61.4)$ \\
1-2 & $9(69.2)$ & $211(38.6)$ \\
3-4 & $9(69.2)$ & $483(88.3)$ \\
Anesthesia type, $n(\%)$ & $4(30.8)$ & $64(11.7)$ \\
Regional & & \\
General & $8(61.5)$ & $201(36.7)$ \\
Operation type, $n(\%)$ & $5(38.5)$ & $346(63.3)$ \\
THA & & \\
TKA & $5(38.5)$ & $119(21.8)$ \\
Comorbidities, $n(\%)$ & $13(100)$ & $436(79.7)$ \\
Diabetes mellitus & $6(46.2)$ & $121(22.1)$ \\
Hypertension & $4(30.8)$ & $26(4.7)$ \\
Ischemic heart disease & $1(7.7)$ & $39(7.1)$ \\
CHF & $6(46.2)$ & $51(9.3)$ \\
COPD & & \\
CRF & & \\
\hline
\end{tabular}

AF, atrial fibrillation; ASA, American Society of Anesthesiologists; COPD, chronic obstructive pulmonary disease; THA, total hip arthroplasty; TKA, total knee arthroplasty; $\mathrm{CHF}$, chronic heart failure; CRF, chronic renal failure.

documented risk factors for mortality in orthopedic surgeries, particularly THA and TKA [15]. General anesthesia has been associated with increased mortality in a study of elderly undergoing hip fracture surgery [16], but it is unclear whether it is the true cause or merely reflects the anesthesiologists' choice for high-risk patients. Hypertension, CHF, and CRF have been implicated as risk factors for AF [17-19], whereas $\beta$-blockers and anticoagulation are among the mainstay of treatment for AF [7]. A higher ASA score is derived from these comorbidities [20].

Several mechanisms may explain the association between new-onset AF following THA or TKA and increased 1-year all-cause mortality. On the one hand, the AF may be a direct cause of mortality due to decreased cardiac output, thromboembolic events or secondary to treatment with rate control, rhythm control, or anticoagulation. On the other hand, the AF may not be directly
Table 3. Multivariate analysis of clinical variables associated with mortality

\begin{tabular}{llll}
\hline Characteristic & $p$ value & Adjusted OR & 95\% CI for OR \\
\hline Age & 0.049 & 1.096 & $1.00-1.201$ \\
General anesthesia & 0.018 & 4.95 & $1.31-18.68$ \\
CHF & 0.029 & 4.76 & $1.18-19.21$ \\
CRF & 0.006 & 5.61 & $1.63-19.34$ \\
\hline
\end{tabular}

OR, odds ratio; $\mathrm{CI}$, confidence interval; $\mathrm{CHF}$, chronic heart failure; CRF, chronic renal failure.

linked but rather a marker of a more complex patient population with more comorbidities.

In our study, both rate control treatment and anticoagulation treatment were associated with increased mortality on univariate analysis, with rate control treatment being statistically significant on multivariate analysis as well. It seems likely that this reflects selection bias as these treatments were presumably utilized for patients with more comorbidities. It is also likely that patients who received rate control treatment had very rapid AF secondary to a poorer clinical condition. Furthermore, in a previous study where post-op AF was associated with increased 1-year mortality rates, the effect was not attenuated by medical treatment for AF [14]. Last, CRF and age were found on multiple analyses to be associated with mortality, making AF more likely to be associated with these risk factors.

Mechanisms by which new-onset AF results in a significant increase in mortality whereas existing AF (PAF/ $\mathrm{CAF}$ ) does not remain unclear. New-onset AF in the perioperative setting may signal new cardiac ischemia, abnormalities of cardiac function, pulmonary embolism, infection, and other factors which may cause death in the short and long term. In contrast, existing AF is associated with numerous comorbidities and thus does not necessarily represent an acute change in the patient's condition. Previous data from the Framingham study show that secondary AF in the community is associated with long-term recurrence and significant cardiovascular morbidity, findings consistent with our study [12]. This is further supported by the finding of increased mortality and other complications in heart failure patients with new-onset $\mathrm{AF}$ as opposed to $\mathrm{PAF}$ and $\mathrm{CAF}$ [21].

Our findings have important clinical implications the appearance of new-onset AF in an elderly patient following a total hip or knee arthroplasty warrants clinical 
Table 4. Characteristics of the AF group

\begin{tabular}{lrlr}
\hline Characteristic & \multicolumn{2}{l}{ Value } & \\
\cline { 2 - 4 } & $\begin{array}{l}\text { alive } \\
(n=270)\end{array}$ & $\begin{array}{l}\text { dead } \\
(n=10)\end{array}$ & $\begin{array}{l}\text { overall } \\
(n=280)\end{array}$ \\
\hline Type of AF, $n(\%)$ & & & \\
PAF & $206(76.3)$ & $3(30)$ & $209(74.6)$ \\
CAF & $31(11.5)$ & $3(30)$ & $34(12.1)$ \\
New onset & $33(12.2)$ & $4(40)$ & $37(13.2)$ \\
Timing of AF, $n(\%)$ & & & \\
Pre & $208(77.0)$ & $4(40)$ & $212(75.7)$ \\
Post & $33(12.2)$ & $4(40)$ & $37(13.2)$ \\
Both & $29(10.7)$ & $2(20)$ & $31(11.1)$ \\
Chronic antiarrhythmic drug treatment & $133(49.2)$ & $3(33.3)$ & $136(48.6)$ \\
In-hospital treatment, $n$ (\%) & & & $49(17.5)$ \\
Treatment with rate control & $43(15.9)$ & $6(60)$ & $58(20.7)$ \\
Treatment with anticoagulation & $53(19.6)$ & $5(50)$ & $33(11.8)$ \\
Treatment with cardioversion & $31(11.5)$ & $2(20)$ & \\
\hline
\end{tabular}

AF, atrial fibrillation; CAF, chronic atrial fibrillation; PAF, paroxysmal atrial fibrillation. * A single patient may require multiple treatments or none at all, as mandated by clinical considerations.

attention, including a careful workup for possible causes and appropriate treatment before discharge. Moreover, diligent follow-up after the hospitalization seems prudent. Since it is unclear whether the postoperative AF is preventable, or whether the AF is directly the cause of death, more studies on the subject are necessary including the optimization of evaluation and treatment for these patients.

The major limitations of the study are its retrospective nature and the relatively small number of new AF events observed. In addition, only all-cause mortality data were available, and it is unclear whether the increased mortality observed was directly related to the AF.

Another limitation is that operations were performed by several different surgeons and assisting residents; however, this represents general orthopedic practice. All surgeons used the same joint prosthesis type and protocol which should limit the chances of bias. Last, one analysis associated the rate control treatment as being statistically significant for mortality instead of the postoperative AF itself. Although this is thought to be a confounder, as treatment is likely to be utilized for patients in poorer clinical condition, the effect of the treatment on mortality cannot be completely excluded.

In conclusion, we demonstrate that 1-year all-cause mortality in elderly patients undergoing total hip replacement or total knee replacement is significantly increased in patients with new postoperative AF. While current guidelines do not specifically address manage-
Table 5. Multivariate analysis of variables associated with mortality in AF group

\begin{tabular}{llll}
\hline Characteristic & $p$ value & $\begin{array}{l}\text { Adjusted } \\
\text { OR }\end{array}$ & \\
\hline When AF type is included & & & \\
$\quad$ CRF & 0.004 & 7.64 & $1.91-30.64$ \\
$\quad$ Type of AF (new onset) & 0.005 & 9.95 & $1.99-49.77$ \\
When AF timing is included & & & \\
$\quad$ CRF & 0.005 & 7.47 & $1.83-30.40$ \\
$\quad$ Timing of AF (post-op) & 0.01 & 7.59 & $1.62-35.62$ \\
\hline
\end{tabular}

$\mathrm{AF}$, atrial fibrillation; OR, odds ratio; CI, confidence interval; CRF, chronic renal failure.

ment of new postoperative AF, given the higher mortality noted in our study, it seems that these patients should at least be carefully monitored for the first year following surgery.

\section{Statement of Ethics}

The study was conducted ethically in accordance with the World Medical Association Declaration of Helsinki. The study protocol was approved by the Hadassah Organization Ethics Committee and was exempt from obtaining written consent from the patients given the study's retrospective design. Reference number 0685-16-HMO. 


\section{Conflict of Interest Statement}

The authors have no conflicts of interest to declare.

\section{Funding Sources}

This research did not receive any specific grant from funding agencies in the public, commercial, or not-for-profit sectors.

\section{Author Contributions}

All authors contributed to data attainment and patient file review. Dr. Varon also summarized the results, performed the statistical analysis, and wrote the article manuscript.

\section{References}

1 Lane NE, Brandt K, Hawker G, Peeva E, Schreyer E, Tsuji W, et al. OARSI-FDA initiative: defining the disease state of osteoarthritis. Osteoarthritis Cartilage. 2011;19(5):478-82.

2 Lawrence RC, Felson DT, Helmick CG, Arnold LM, Choi H, Deyo RA, et al. Estimates of the prevalence of arthritis and other rheumatic conditions in the United States, Part II. Arthritis Rheum. 2008;58:26-35.

3 Zhang W, Moskowitz RW, Nuki G, Abramson $\mathrm{S}$, Altman RD, Arden N, et al. OARSI recommendations for the management of hip and knee osteoarthritis, Part II: OARSI evidencebased, expert consensus guidelines. Osteoarthritis Cartilage. 2008;16(2):137-62.

4 Kurtz S, Ong K, Lau E, Mowat F, Halpern M. Projections of primary and revision hip and knee arthroplasty in the United States from 2005 to 2030. J Bone Joint Surg Am. 2007; 89(4):780-5.

5 Inacio MCS, Dillon MT, Miric A, Navarro RA, Paxton EW. Mortality after total knee and total hip arthroplasty in a large integrated health care system. Perm J. 2017;21:16-171.

6 Lloyd-Jones DM, Wang TJ, Leip EP, Larson MG, Levy D, Vasan RS, et al. Lifetime risk for development of atrial fibrillation: the Framingham Heart Study. Circulation. 2004; 110(9):1042-6.

7 Falk RH. Atrial fibrillation. N Engl J Med. 2001;344(14):1067-78.

8 van Diepen S, Bakal JA, McAlister FA, Ezekowitz JA. Mortality and readmission of patient with heart failure, atrial fibrillation, or coronary artery disease undergoing noncardiac surgery: an analysis of 38,047 patients Circulation. 2011;124:289-96.
9 Long G, Suqin S, Li G, Weihong Y, Zhenhu $W$. Impact of atrial fibrillation on postoperative outcomes after total knee arthroplasty-A retrospective study. J Orthop Sci. 2016 Sep; 21(5):652-7.

10 Khormaee S, Do HT, Mayr Y, Gialdini G, Kamel H, Lyman S, et al. Risk of ischemic stroke after perioperative atrial fibrillation in total knee and hip arthroplasty patients. J Arthroplasty. 2018 Sep;33(9):3016-9.

11 Tao L, Xiaodong X, Fan L, Gang D, Jun D. Association between new-onset postoperative atrial fibrillation and 1-year mortality in elderly patients after hip arthroplasty. Aging Clin Exp Res. 2020 May;32(5):921-4.

12 Lubitz SA, Yin X, Rienstra M, Schnabel RB, Walkey AJ, Magnani JW, et al. Long-term outcomes of secondary atrial fibrillation in the community: the Framingham Heart Study. Circulation. 2015;131(19):1648-55.

13 Brathwaite D, Weissman C. The new onset of atrial arrhythmias following major noncardiothoracic surgery is associated with increased mortality. Chest. 1998;114(2):462-8.

14 Leibowitz D, Abitbol C, Alcalai R, Rivkin G, Kandel L. Perioperative atrial fibrillation is associated with increased one-year mortality in elderly patients after repair of hip fracture. Int J Cardiol. 2017;227:58-60.

15 Bhattacharyya T, Iorio R, Healy WL. Rate of and risk factors for acute inpatient mortality after orthopaedic surgery. J Bone Joint Surg Am. 2002;84-A(4):562-72.
16 Chu CC, Weng SF, Chen KT, Chien CC, Shieh JP, Chen JY, et al. Propensity score-matched comparison of postoperative adverse outcomes between geriatric patients given a general or a neuraxial anesthetic for hip surgery: a population-based study. Anesthesiology. 2015;123(1):136-47.

17 Krahn AD, Manfreda J, Tate RB, Mathewson FA, Cuddy TE. The natural history of atrial fibrillation: incidence, risk factors, and prognosis in the Manitoba Follow-Up Study. Am J Med. 1995;98(5):476-84.

18 Santhanakrishnan R, Wang N, Larson MG, Magnani JW, McManus DD, Lubitz SA, et al. Atrial fibrillation begets heart failure and vice versa: temporal associations and differences in preserved versus reduced ejection fraction. Circulation. 2016;133(5): 484-92.

19 Watanabe H, Watanabe T, Sasaki S, Nagai K, Roden DM, Aizawa Y. Close bidirectional relationship between chronic kidney disease and atrial fibrillation: the Niigata preventive medicine study. Am Heart J. 2009;158:62936.

20 Dripps RD. New classification of physical status. Anesthesiol 1963;24:111.

21 Mogensen UM, Jhund PS, Abraham WT, Desai AS, Dickstein K, Packer M, et al. Type of atrial fibrillation and outcomes in patients with heart failure and reduced ejection fraction. J Am Coll Cardiol. 2017;70(20):2490500 